\section{Screening Volunteers}

Volunteer screening supports the process of educating volunteer applicants about Virginia Cooperative Extension programs and creates the opportunity to allow faculty and staff to learn more about the interests of the volunteer applicant. The screening process also helps to protect the wellbeing of Virginia Cooperative Extension programs, its participants, and volunteers.

\section{Volunteer Screening Levels}

\section{High-Level Screening Positions}

High-level screening positions are determined based on the level of risk associated with the volunteer position and the potential for loss. These positions include:

- 4-H Volunteers that provide direct supervision to 4-H youth programs, handle 4-H club funds, or transport youth;

- 4-H Camp Volunteers 18 years of age and older (must be completed annually);

- Master Volunteers

- Volunteers in Positions of Trust (working with youth, seniors, those with disabilities, handling funds, or unsupervised access to VCE equipment/facilities).

\section{Low-Level Screening Positions}

Collaborative/Occasional/One-Time Volunteers These volunteer positions are under constant/ direct supervision of a VCE faculty/staff member or designated volunteer, or any person, who, as a part of their normal compensated employment, assists VCE in the accomplishment of a shared goal (teachers, Parks and Recreation staff, etc). No interview, reference check, or criminal background screening is required.

\section{Contact}

There are many ways you can get involved with us. If you would like specific information on a particular volunteer opportunity, or, if you are interested in volunteering but not sure in what way, contact your local Extension office.

\section{www.ext.vt.edu/offices}

For more information about Virginia Cooperative Extension's volunteer efforts, contact:

\section{Joi Saville}

4-H Operations \& Events Coordinator 540-231-6372

jdyer@vt.edu

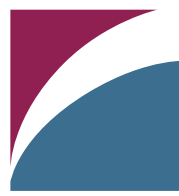

Virginia Cooperative Extension

Virginia Tech • Virginia State University

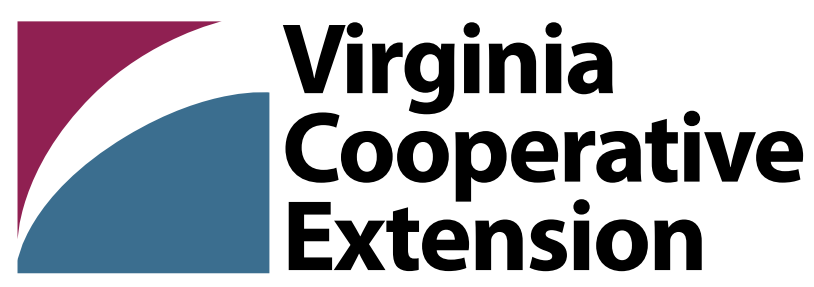

Virginia Tech • Virginia State University

\section{Virginia Cooperative Extension VOLUNTEERS}

Extending Knowledge and Improving Lives

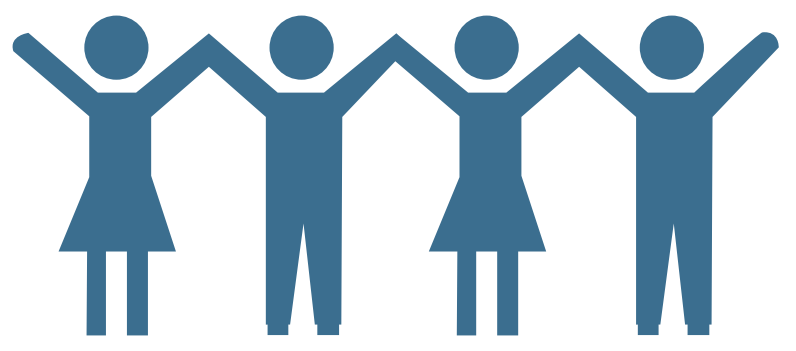

Extension volunteers ...

Educate and engage the public

Develop community through social connections

Serve as knowledgeable and credible resources

Advocate for Virginia Cooperative Extension

Are prepared leaders

Volunteer Screening Guidelines Adopted 2014 


\section{Screening Process}

There are four primary steps to complete the volunteer screening process for Virginia Cooperative Extension high-level screening volunteer positions:

- Application/Enrollment Form - submit a completed application to the local unit office.

- Interview - a faculty/staff member or currently enrolled volunteer will make personal contact with the applicant to explore interests, skills, and to determine fit of the applicant with the volunteer position.

- Reference Checks - up to three references may be contacted by phone, email, or mail to provide information about the applicant's professional, personal, and community experience.

- Criminal Background Screening* - National Criminal Index Search, National Sex Offender Registry Search, Name, SSN, and DOB validation with address history verification, County or Statewide Level Criminal Search. * Repeated every three years (annually for 4-H camp volunteers or any volunteers involved in an overnight event/activity with youth).

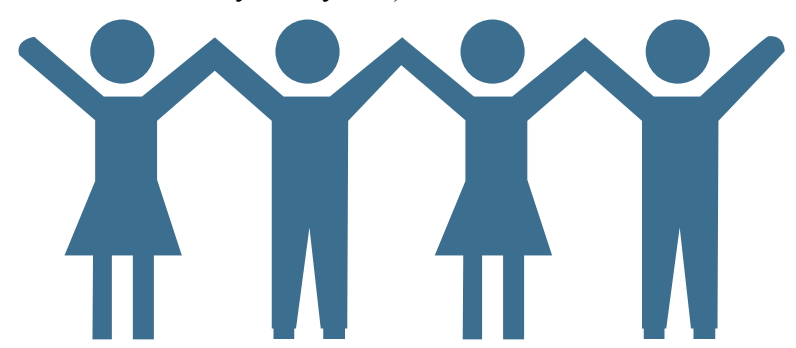

\section{PreSearch Provider}

Since 1995, PreSearch Background Services has performed over two million employment screening background checks. PreSearch Background Services uses a secure, online, system to collect information from volunteer applicants to complete the criminal background screening. Personal information is entered into the system directly by the applicant and Virginia Cooperative Extension does not have access to social security numbers provided by the applicant. Results of the screening are only visible to the VCE State Administrators and are not released to local unit offices in order to protect the applicant's confidentiality.

\section{Cost}

The cost for the search is $\$ 9.95$ and the applicant can contact the local VCE unit for details on payment information.

\section{Accepting Outside Criminal Background Screenings}

Applicants may submit outside screening results that meet the scope of the search and have been completed within three years directly to the State Office for review.

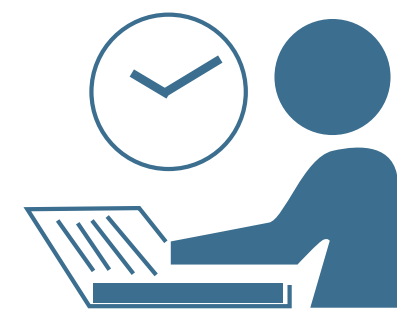

\section{Recommended Criteria for Exclusion}

Virginia Cooperative Extension will utilize the best practices and guidelines from the National Recreation and Park Association publication, Recommended Guidelines for Credentialing Volunteers, to determine a volunteer applicant's eligibility to serve. Many public and private organizations have adopted these guidelines.

A person should be disqualified and prohibited from serving as a volunteer if the person has been found guilty of the following crimes within the specified time.

\section{Sex Offenses}

- All Sex Offenses - Regardless of the amount of time since offense Examples include: child molestation, rape, sexual assault, sexual battery, sodomy, prostitution, solicitation, indecent exposure, etc.

\section{Felonies}

- All Felony Violence - Regardless of the amount of time since offense. Examples include: murder, manslaughter, aggravated assault, kidnapping, robbery, aggravated burglary, etc.

- All Felony offenses other than violence or sex within the past 10 years. Examples include: drug offenses, theft, embezzlement, fraud, child endangerment, etc.

\section{Misdemeanors}

- All Misdemeanor Violence offenses within the past seven years. Examples include: simple assault, battery, domestic violence, hit and run, etc.

- All misdemeanor drug and alcohol offenses within the past five years or multiple offenses in the past 10 years. Examples include: driving under the influence, simple drug possession, drunk and disorderly, public intoxication, possession of drug paraphernalia, etc.

- Any other misdemeanor within the past five years that would be considered a potential danger to children or is directly related to the functions of that volunteer. Examples include: contributing to the delinquency of a minor, providing alcohol to a minor, theft if person is handling monies, etc.

This recommendation does not apply if criminal charges resulted in acquittal, Nolle Prosse, or dismissal It is recommended the any who has been chared for any of the disqulifying offenses or for cases pending in cout auld not be permitted to volunter until the official adjudication of the case. 